\title{
A Portable and Compact 3D Body Scanner --- 3D Body Scanners Traveling Type ---
}

\author{
Masaki HAYASHI*a,b ${ }^{\text {, Hideto KAMESHIMA }}{ }^{a}$, Yuji NISHIO ${ }^{a}$, Yukio SATO ${ }^{a, b}$ \\ ${ }^{a}$ Spacevision,Inc., Tokyo, Japan; \\ ${ }^{\mathrm{b}}$ Keio University, Kanagawa, Japan
}

\section{Keywords: 3d body scanning, portable, high-speed, smart calibration}

Introduction: A portable, ultra-compact, and high-speed scanners for 3D human body scanning is described in this paper. The 3D Body Scanner Traveling Type, shown in figure.1, is an unprecedented "portable" 3D scanning system which can be disassembled into nine portable projector-camera components.

Spacevision is a leading company for portable 3D human body scanner, which is called "Cartesia 3D Body Scanner", which is a separable and portable system. In this paper, we present our new "separable and easy to carry out" $3 d$ body scanner, which is called," Traveling Type". The portability of it must cultivate new uses of $3 d$ body scanning technologies.

System: Our system consists of three pole units (Typically. Four pole-units type is also available). Each pole unit has three vertically stacked projector-camera components and a pedestal (see figure.1). The dimensions of each projector-camera component are $7 \mathrm{~cm}$ width, $20 \mathrm{~cm}$ depth and $65 \mathrm{~cm}$ height. The total height of the assembled pole unit is about $200 \mathrm{~cm}$. The weight of each component is only 3.1 $\mathrm{kg}$, so that it can be carried separately with a suitcase by one person.

Portability and Compactness: Portability for 3d body scanners had been highly demanded from several kinds of industries for long, but never achieved before. Our system realizes a great portability and compactness with two following solutions:

(1) Decomposable into portable units.

As already mentioned before, each pole of our system can be disassembled into 3 modules, and each module is easy to carry. Therefore, users can easily move or transport the whole system to another place by disassembling it into 9 parts.

(2) Adjustable with multi-camera calibration whenever you replace your system.

Multi-camera calibration must be done by users after placing all pole units onto their site. Namely, each projector-camera modules has its own projector-camera parameters calculated before shipments, so all you have to do is just perform multi-camera calibration, which only takes about 5 minutes, after placing the system onto your site. This calibration procedure enables you to replace the system to another site as many as you like. We strongly believe that this portability can extend how and where to use $3 d$ body scanners.

High Speed Capture with colored texture: The system measures the 3D human body shape and its color (see figure 2) within 2.0 seconds owing to its fast capturing of a range image. This high speed capturing is derived from multi-camera calibration performed in advance. Measurement density can be increased up to 1 million points or more with keeping the average margin of error within $3 \mathrm{~mm}$ or less.

Easy setup: Setup is also easy for even an unskilled operator. First, the bottom projector-camera component is placed on the pedestal and fastened by a wrench. Next, the middle component and upper component are laid on the bottom component in order. The viewpoints of the nine projector-cameras in each pole units are arranged beforehand in order to measure the human body with less occluding parts. Careful or precise placement of the pole units is not needed because calibration process assures the accuracy of the synthesis of the data taken from each pole units. Moreover, the calibration procedure of projector-camera parameters is quite simple and finishes quickly.

* hayashi@space-vision.jp ; $\quad$ +81- 3- 5428-4144; www.space-vision.jp 

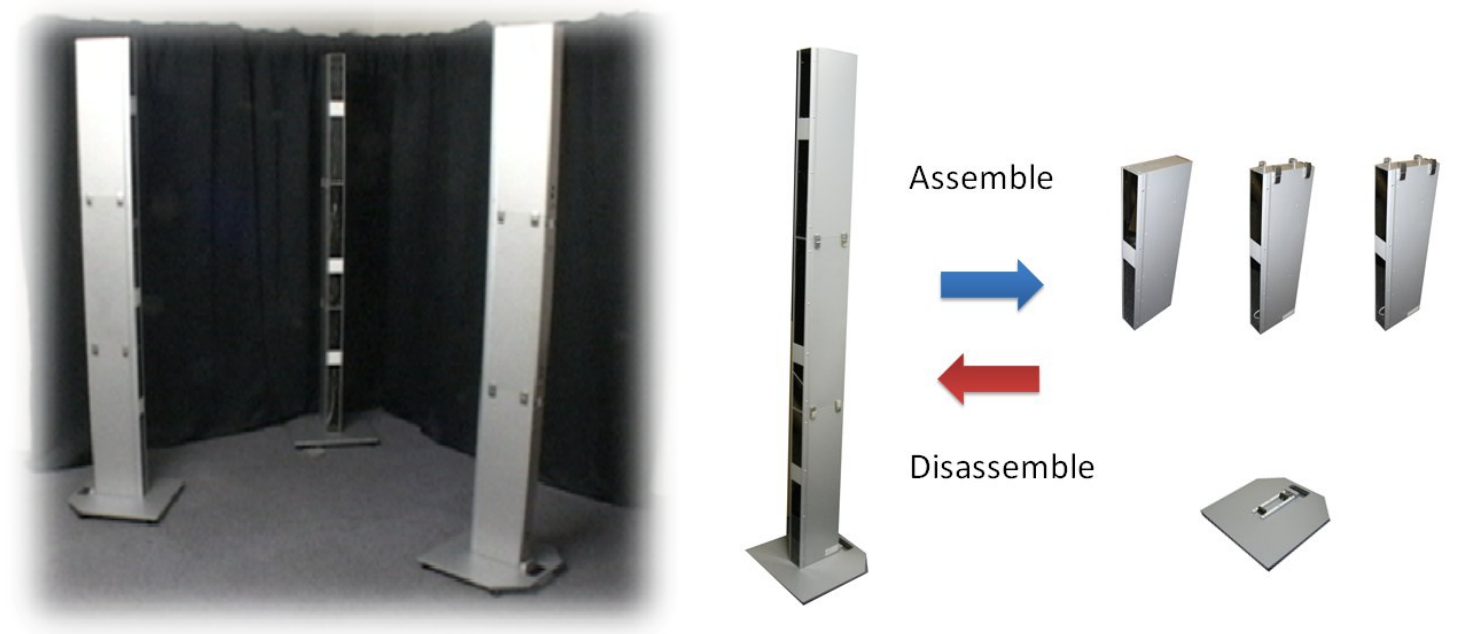

Disassemble

Fig.1. Components of Portable 3D Body Scanner. Left: The whole system. Right: Each pole can be disassembled into 3 pro-cam modules. You just need to stack up modules to (re-)assemble them.
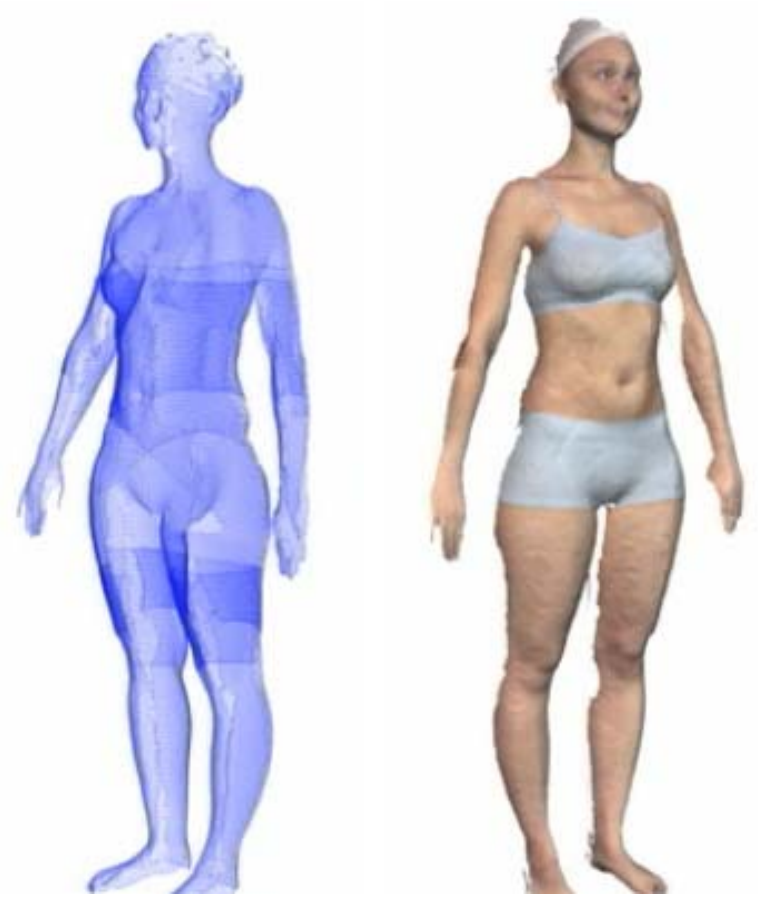

Fig.2. Measured Human Body. Left: Point cloud without texture. Right: Textured point cloud. 